\title{
Forward and inverse dynamic study during pedaling: Comparison between the young and the elderly
}

\author{
Jeongwoo Seo ${ }^{\mathrm{a}}$, Jinseung Choi ${ }^{\mathrm{a}, \mathrm{b}}$, Dongwon Kang ${ }^{\mathrm{a}}$, Seungtae Yang ${ }^{\mathrm{a}}$, Daehyeok Kim ${ }^{\mathrm{a}}$ and \\ Gyerae Tack ${ }^{\mathrm{a}, \mathrm{b}, *}$ \\ ${ }^{\mathrm{a}}$ Department of Biomedical Engineering, College of Biomedical \& Health Science, Konkuk University, \\ Chungju, Korea \\ ${ }^{\mathrm{b}}$ BK21 Plus Research Institute of Biomedical Engineering, Konkuk University, Chungju, Korea
}

\begin{abstract}
.
BACKGROUND: As it is not easy to investigate various variables that affect exercise efficacies and cause injuries while pedaling in the actual experiment, especially for the elderly, the musculoskeletal model simulation with a comparison of measured electromyography (EMG) data could be used to minimize experimental trials.

OBJECTIVE: The measured EMG data were compared with the muscle activities from the musculoskeletal model through forward (FD) and inverse dynamic (ID) analysis.

METHODS: EMG was measured from eight young adult (20's) and eight elderly (70's) in three minutes pedaling with a constant load and 40 revolutions per minute (RPM) cadence. The muscles used for the analysis were the VastusLateralis, Tibialis Anterior, Bicep Femoris, and Gastrocnemius Medial. Pearson's correlation coefficients of the muscle activity patterns, on-off set, and peak timing at the maximum muscle activity were calculated and compared. BIKE3D and GaitLowerExtremity model were used for the FD and ID simulation.

RESULTS: There are significant correlations in the muscle activity patterns except in the case of Biceps Femoris muscle by ID. Thus, it can be concluded that muscle activities of model \& EMG showed similar results.

CONCLUSION: The result shows that it could be possible to use the musculoskeletal model for various pedaling simulations.
\end{abstract}

Keywords: Musculoskeletal modeling, electromyography, pedaling, aging

\section{Introduction}

Cycling helps strengthen the muscular power of the lower body and the cardiopulmonary function, thus improving the physical strength of the riders [1]. However, improper pedaling posture and load can increase the possibility of injury occurrence. The variables that can cause injuries and affect exercise efficacies while pedaling are diverse such as the seat tube angle, saddle height, and pedal arm length. To investigate the influences of all variables on the pedaling, repeated experiments by changing each variable are necessary. However, it is difficult to do consistent experiments in order to obtain meaningful results. Therefore, it is necessary to simulate various controlled variables before the actual experiment

\footnotetext{
${ }^{*}$ Corresponding author: Gyerae Tack, Department of Biomedical Engineering, College of Biomedical \& Health Science, Konkuk University, 322 Danwoldong, Chungju, Korea. E-mail: grtack@kku.ac.kr.
} 
Table 1

Anthropometric data of the subjects and the characteristics of their cycle (mean $\pm \mathrm{SD}$ )

\begin{tabular}{ccccccc}
\hline Group & Age (year) & Weight $(\mathrm{kg})$ & Height $(\mathrm{cm})$ & Thigh $(\mathrm{cm})$ & Tibia $(\mathrm{cm})$ & Foot $(\mathrm{cm})$ \\
\hline 20 's & $26.3 \pm 0.7$ & $65.2 \pm 5.5$ & $174.3 \pm 4.1$ & $85.3 \pm 4.1$ & $37.0 \pm 2.8$ & $240.3 \pm 2.2$ \\
70 's & $76.4 \pm 5.1$ & $41.9 \pm 5.0$ & $163.3 \pm 4.6$ & $41.9 \pm 1.1$ & $39.5 \pm 1.1$ & $259.7 \pm 0.6$ \\
\hline Group & Inseam $(\mathrm{cm})$ & Upper arm $(\mathrm{cm})$ & Forearm $(\mathrm{cm})$ & Saddle position $(\mathrm{cm})$ & Handle position $(\mathrm{cm})$ & Pedal length $(\mathrm{cm})$ \\
\hline 20 's & $80.4 \pm 3.6$ & $30.5 \pm 0.8$ & $28.8 \pm 1.5$ & $17.7 \pm 1.1$ & $66.5 \pm 1.1$ & $10.7 \pm 0.0$ \\
70 's & $71.8 \pm 4.3$ & $27.8 \pm 1.5$ & $25.5 \pm 1.7$ & $22.0 \pm 1.3$ & $61.3 \pm 1.3$ & $10.7 \pm 0.0$ \\
\hline
\end{tabular}

and to get various expected results with a minimum number of the experiment.The musculoskeletal model simulation with a comparison of measured EMG data could be used to overcome such difficulties. There are two major approaches to doing musculoskeletal model simulation. The first is the forward dynamic (FD) method, which requires only the dimension of the cycle and anthropometric data of the individual without actual experiment to predict the results. However, the results of FD can be limited due to the difference of the individual in muscle power and kinematic characteristics. The second is the inverse dynamic (ID) method, which requires results of the actual experiment. The results of ID could show relatively accurate results compared with those of FD, and identify additional various biomechanical properties such as joint torques and contact forces that are difficult to measure. Musculoskeletal models have been applied to the study of pedaling. Rasmussen et al. [2] used the ID to study the changes in the tendon energy of the lower limb muscles and the foot angles according to the changes in the tendon elasticity values while pedaling. Shin et al. [3] used the FD to compare muscle activity patterns of simulation with actual EMG measurement while pedaling. However, there has been a lack of studies on cycle fitting and on the elderly using a musculoskeletal model with the changes of various pedaling variables. Since the elderly has less muscle power and exercise function than the young, repeated experiment that requires controlling of pedaling power and RPM can be limited. Those are the reasons why the pedaling study on the elderly is insufficient. In general, the elderly showed less endurance, muscle power, flexibility, balance, and response time than the young [4]. It means that there will be differences in biomechanical results between the young and the elderly while pedaling. Thus, this study compared the results of FD and ID simulation of the musculoskeletal model with the measured muscle activities of the young and the elderly while pedaling.

\section{Method}

\subsection{Subject}

The study was conducted on eight male adults in their twenties and eight elderly in their seventies who are healthy without the history of musculoskeletal diseases and can walk normally. The protocol for this study was confirmed from the ethics committee of the University. Before the experiment, experimental procedures were explained to all subjects, and written informed consent was received. The characteristics of the subjects were shown in Table 1.

\subsection{Experimental}

Three-dimensional motion analysis system with six infrared cameras (Motion Analysis Corp., USA) and pedal force plate (Dacell Corp, Korea) were used to acquire the motion and pedal reaction force data with $120 \mathrm{~Hz}$ and 1,200 Hz sampling frequency, respectively. Twenty-three reflective markers were 

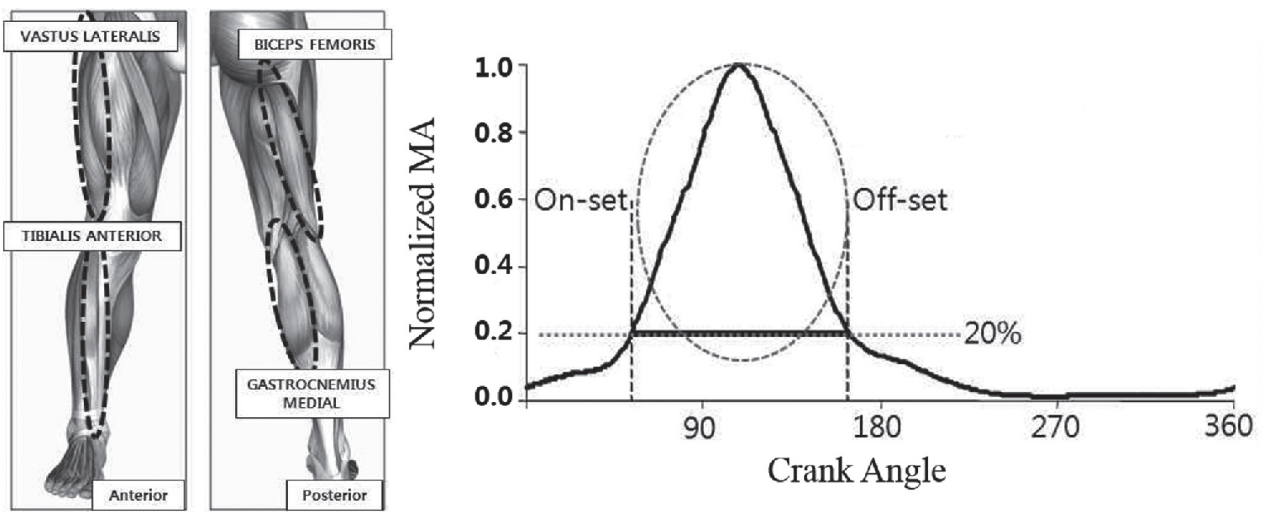

Fig. 1. Four muscles and the definition of on-off set timing with Normalized MA.

attached to the plugin set, and the pedaling was conducted for 3 minutes and with 40 RPM cadence (Fig. 1). For the measurement of muscle activities, the bipolar surface wireless EMG system (Trigno wireless EMG system, Delsys Inc., USA) with the sampling frequency of 1,200 $\mathrm{Hz}$ was used with $8 \mathrm{~mm}$ diameter $\mathrm{Ag} / \mathrm{AgCl}$ electrode and a $10 \mathrm{~mm}$ inter-electrode distance. The muscle activities were measured at mono-articular muscles (Vastus Lateralis (VL) and Tibialis Anterior (TA)) and multi-articular muscles (Biceps Femoris (BF) and Gastrocnemius Medial (GM)), which are mostly used during pedaling.

\subsection{Data analysis}

Totally two-minutes data which excluded the initial and final 30 seconds were used for the analysis. For a comparison between the results from musculoskeletal model simulation and measured EMG data, data were expressed as the normalized muscle activities (Normalized MA) as shown in Fig. 1, and its repeatability and reliability were checked [5]. The muscle activity was defined as the summation of all muscle activities by excluding less than $20 \%$ muscle activity values as shown in Fig. 1. A secondorder zero-delay Butterworth filter with $10 \mathrm{~Hz}$ cutoff frequency was used as a low-pass filter for noise removal of motion data [6]. EMG data were obtained through band-pass filtering with $15-500 \mathrm{~Hz}$ by using a fourth-order zero delay Butterworth filter. All calculation was carried out with Matlab R2013a (Mathworks Inc., USA) software. The normality was tested by checking the normal distribution of EMG data, and then Pearson's correlation coefficient $(\alpha=0.05)$ was calculated by SPSS 19.0 (SPSS Inc., USA).

\subsection{Musculoskeletal model simulation}

The Anybody (AnyBody Technology, Denmark) software is used for musculoskeletal model analysis in this study. The simulation model is GaitLowerExtremity based on the Hill-type model. This model is an enhanced accurate lower extremities model that has 56 muscles divided into 176 branches. This study only considers the muscles ( 31 muscles, 115 branches) attached at the femur [7]. First, measured information of the subjects (their height, weight, inseam and the length of the thigh, tibia, upper arm and forearm, hand and foot sizes, pelvic width, and positions of saddle and handle, pedal arm length and width measured by the reference [8]) was applied to the BIKE3D FD model on the Anybody. Second, muscle activities of all subjects were calculated by the optimization of ID model after 3D motion and pedal reaction force data of 16 subjects were imported and scaled into GaitLowExtremity model. 
Table 2

Correlation coefficient between EMG and simulation $\left({ }^{*} p<0.05\right)$

\begin{tabular}{cccccc}
\hline \multicolumn{2}{c}{ Muscle } & VL & TA & BF & GM \\
\hline \multirow{2}{*}{20 's } & FD & $0.630^{*}$ & $0.960^{*}$ & $0.503^{*}$ & $0.839^{*}$ \\
& ID & $0.778^{*}$ & $0.702^{*}$ & -0.081 & $0.675^{*}$ \\
70 's & FD & $0.941^{*}$ & $0.293^{*}$ & $0.771^{*}$ & $0.784^{*}$ \\
& ID & $0.750^{*}$ & $0.754^{*}$ & $0.394^{*}$ & $0.958^{*}$ \\
\hline
\end{tabular}

\section{Results}

Figure 2 shows measured EMG, and simulated muscle activities of ID and FD by the crank angle. For the young adult, there was a significant correlation among measured EMG, results by ID and FD except the result of BF by ID. For the elderly, there was a significant correlation among measured EMG, results by ID and FD for all muscles (Table 2).

\section{Discussion and conclusion}

In this study, muscle activities (EMG) of the young adult in their twenties and the elderly in their seventies while pedaling are compared with those of a musculoskeletal model by FD and ID for the application of cycle pedaling. Totally 16 subjects (eight young and eight old) participated in the cycle pedaling, and ID and FD were performed by using measured 3D motion, pedaling force and subjects' anthropometric data.

Results indicated that FD could not show the differences in muscle force due to the age, which constitutes its major disadvantage. Since FD used a standard musculoskeletal model with arbitrary forces, there existed a limitation in the representation of the characteristics of the individual subject. However, the results of ID showed similar patterns with measured EMG since ID uses measured pedal force and kinematic data. Therefore, it is better to use ID for the representation of the characteristics of the age difference.

The biggest difference in EMG between the young and the elderly was the usage of TA muscle. As the twenties used TA muscle efficiently by activating at the top-dead-center where the pedal reaches the highest, the seventies used it inefficiently by activating at the point where the vertical pedaling occurred. This is due to that the ankle movement of the elderly is not as freely as that of the young. Thus the inefficient use of TA muscle for the elderly occurred during pedaling. Aging has a profound effect on muscle fiber type distribution and properties, therefore decreasing the efficiency of the force [9]. This explains the slight difference in muscle activity between the young and the elderly during pedaling. For the young and the elderly, there were significant correlations in the muscle activity patterns except in the case of BF muscle by ID. In the case of BF muscle by ID, for the twenties there did not exist significant correlation, which requires an additional study to explain. In the cases of GM and VL muscle, the correlation was significant. This is due to the fact that the characteristics of the musculoskeletal model in FD and ID with experiment data were optimized properly by the subjects' anthropometric data [10].

The on, off-set and peak timing were slightly different, but made no direct effect on the correlation of muscle pattern. In the case of multi-articular muscles such as BF and GM, precise control of the movement is carried out by involving more than single joint. However, due to straight-lined muscles in the musculoskeletal model, the bending of the muscles in the model must be considered during joint movement in the future [10]. The complex interaction among those factors induced the difference in on, off-set, and peak timing at the maximum muscle activities and the integrated muscle activities [11]. 



Fig. 2. EMG, simulation results of FD and ID. 
Therefore, it is necessary to modify slightly the musculoskeletal model in this study, which is suitable for the young and the elderly, for the identification of muscle activation by on, and off-set timing. Since all muscle activity patterns showed a significant correlation in both FD and ID, the disadvantage of a limited number of EMG channels can be supplemented.With FD, without actual experiment, muscle patterns can be calculated by using the dimension of the cycle and the subjects' anthropometric data. However, FD is limited in investigating the effects of aging compared with ID. The results of muscle activity patterns showed significant correlation, and on, off-set and peak timing were slightly different, however, not similar enough to make an effect on muscle activity patterns. Yet there is improvement room in the future study: the bending of the muscles in the model must be considered due to straightlined muscles in the musculoskeletal model [10]; it is necessary to increase the number of subjects for the characterization of individual muscle.

This study compares the results of FD and ID simulation of the musculoskeletal model with the measured muscle activities of the young and the elderly while pedaling. The comparisons of the actual experiments with the simulation results show significant correlations in the muscle activity patterns except in the case of BF muscle by ID. Thus, it can be concluded that muscle activities of model and EMG show similar results. The result of this study shows that it could be possible to use the musculoskeletal model for various pedaling simulation.

\section{Acknowledgement}

This work was supported by Konkuk University.

\section{References}

[1] Kim IK, Jung MH. The effect of ride a bicycle on the physical fitness of an old person, Journal of Physical Education \& Sports Science, 1996; 14(1): 115-124.

[2] Rasmussen J, Damsgaard M, Christensen ST. Simulation of tendon energy storage in pedaling, MEDICON 2001: Proceedings of the international federation for medical \& emp; Biological engineering, 2001; 4: 7391-7395.

[3] Shin YH, Choi JS, Kang DW, Seo JW, Lee JH, Kim JY, et al. A study on human musculoskeletal model for cycle fitting: comparison with EMG, International Journal of Medical, Health, Pharmaceutical and Biomedical Engineering, 2015; 9: 43-47.

[4] McCartney N, Hicks AL, Martin J, Webber CE. Long-term Resistance Training in the Elderly: Effects on Dynamic Strength, Exercise Capacity, Muscle, and Bone, The Journal of Gerontology: Biological Sciences, 1995; 50(2): 97-104.

[5] Albertus-Kajee Y, Tucker R, Derman W, Lambert M. Alternative methods for normalizing EMG during cycling, Journal of Electromyography and Kinesiology, 2010; 20(6): 1036-1043.

[6] Brian SB, Li L. Lower extremity muscle activities during cycling are influenced by load and frequency, Journal of Electromyography and Kinesiology, 2003; 13(2): 181-190.

[7] Seo JW, Kang DW, Kim JY, Yang ST, Kim DH, Choi JS, Tack GR. Finite element analysis of the femur during stance phase of gait based on musculoskeletal model simulation, Bio-Medical Materials and Engineering, 2014; 24(6): 24852493.

[8] Mestdagh K. Personal perspective: in search of an optimum cycling posture, Applied Ergonomics, 1998; 29(5): 325-334.

[9] Sacchetti M, Lenti M, Di Palumbo AS, De Vito G. Different effect of cadence on cycling efficiency between young and older cyclists, Medicine \& Science in Sports \& Exercise, 2010; 42(11): 2128-2133.

[10] Damsgaard M, Rasmussen J, Christensen ST, Surma E, Zee M. Analysis of musculoskeletal systems in the AnyBody modeling system, Simulation Modelling Practice and Theory, 2006; 14(8): 1100-1111.

[11] Jacobs R, Bobbert MF, van Ingen Schenau GJ. Function of mono- and biarticular muscles in running, Medicine and Science in Sports and Exercise, 1993; 25(10): 1163-1173. 